\title{
Effects of Quantum well Size Alteration on Excitonic Population Oscillation Slow Light Devices Properties
}

\author{
Hassan Kaatuzian, Hossein Shokri Kojori, Ashkan Zandi, Reza Kohandani \\ Photonics Research Laboratory (PRL), Department of Electrical Engineering, Amirkabir University of Technology \\ (Tehran Polytechnic), Tehran, Iran \\ Email: hsnkato@aut.ac.ir
}

Received 2013

\begin{abstract}
This paper investigates the effects of quantum well size changes on center frequency and slow down factor of an slow light device. In this way, we consider the quantum well size alteration effects on oscillator strength and binding energy of exciton. First, we investigate the variations in oscillator strength of exciton due to different quantum well size. Second, exciton binding energy level shift due to size of quantum well is investigated. According to this analysis, we have developed a new method for tuning slow light device bandwidth center frequency and slow down factor. Analysis and simulation of a basic GaAs/AlGaAs quantum wells optical slow light device based on excitonic population oscillation shows that size of quantum wells could tune both of the frequency properties and slow down factor of an optical slow light device. In our simulation with 34 quantum wells each with the width of $60 \AA$, we have received the slow down factor of more than 60,000. These achievements are useful in optical nonlinearity enhancements, all-optical signal processing applications and optical communications.
\end{abstract}

Keywords: Slow Light; Coherent Population Oscillation; Quantum Well; Exciton Oscillator Strength; Exciton Binding Energy

\section{Introduction}

During past years several experiments have shown the possibility of controlling the speed of light [1]. Optical buffers have lots of potential scientific and engineering applications [2].

The applications of such an optical buffers (OB) could be 1) bit level synchronization; 2) data packet synchronization; 3) Optical gates and 4) boosting nonlinear interaction [3-4-5]. There are several methods to slow down the speed of light. Each of these methods are different in their mediums and principles. Slow light could be realized by methods such as electromagnetically induced transparency (EIT) in an ultra-cold atomic cloud, waveguide in photonic crystals, coupled resonator optical waveguide [6-7]. By considering the principle of the slow light, these techniques are categorized in WG dispersion and material dispersion [3]. In this paper, our focus will be on material dispersion.

For achieving the slow light in semiconductors, large material dispersion should be obtained. For this goal, coherent population oscillation (CPO), stimulated Brillouin and Raman scattering, four wave mixing (FWM) and EIT can be used [1-5]. There are many research reports about semiconductor optical buffer because of their advantages in compactness, integration with other optical devices, ability of tuning the buffer parameters and wide range of working temperature. Recently, there are huge uses of CPO and FWM in quantum well (QW) and quantum dot (QD) semiconductor to change in absorption or gain spectra [2-8].

The excitonic population oscillation has been successfully presented by $\mathrm{Ku}$ et al [1]. The experimental setup consists of a linearly polarized pump tuned to the resonance energy of 1s heavy-hole $(\mathrm{HH})$ exciton in quantum wells which is incident in the QW growth direction, as shown in Figure 1. A weak signal with parallel or orthogonal polarization to the pump propagates along QW. Pump causes variation of absorbance and phase delay [9]. Dephasing mechanisms exhibit observable features in absorption spectra. However the effect of excitation induced dephasing and spin flip should be considered in formulation for the dephasing of population in semiconductor quantum structure under intense pump. According to these considerations, slow light base on excitonic population has shown in [1].

There could be several choices to control an optical buffer. These control procedures can be divided in two sections. The first one is before the fabrication and the second is after the fabrication. Before fabrication, the techniques such as changing the QW geometry, the dop- 
ing, the alloy percentage and the materials of QW could be employed to design a practical optical buffer [7]. After fabrication, current injection and pump intensity are the choices for tuning of OB. Pump intensity change the Rabi frequency of QW material and the population of carriers; so, these parameters affect the profile of absorption spectra which could change the steep of refractive index in resonance frequency [5].

In this paper, the effects of changing quantum well size on tuning slow device properties will present. Considering the effect of change in quantum well width and dielectric constant mismatch on binding energy and oscillator strength, we developed a new technique for controlling the slow dawn factor $\left(\mathrm{SDF}=c / v_{g}\right)$ and the bandwidth center frequency. According to simulations, for the constant effective length of QWs, decreasing in QW width and as a result increasing in the number of QWs will cause two effects. First one is slow down factor enhancement. So, increasing in oscillator strength of exciton will improve the slow down factor. Second effect is enhancement in binding energy and downshift of center frequency. So, increasing in binding energy of exciton will shift frequency response of a quantum well slow light device in frequency domain.

This paper is organized as follows. In section 2 theory of slow light via excitonic population oscillation, the effect of quantum well width on oscillator strength and binding energy of excitons are presented in three subsections. Section 3, shows the modified model for description of a slow light device to investigate oscillator strength effects. The results and discussion is presented in section 4, also in this section we present our new tuning technique. Section 5, concludes the paper.

\section{Theory}

Figure 1 shows typical schematic of a multiple quantum wells (MQW) structure which is contained pump and probe signal for slow light purpose.

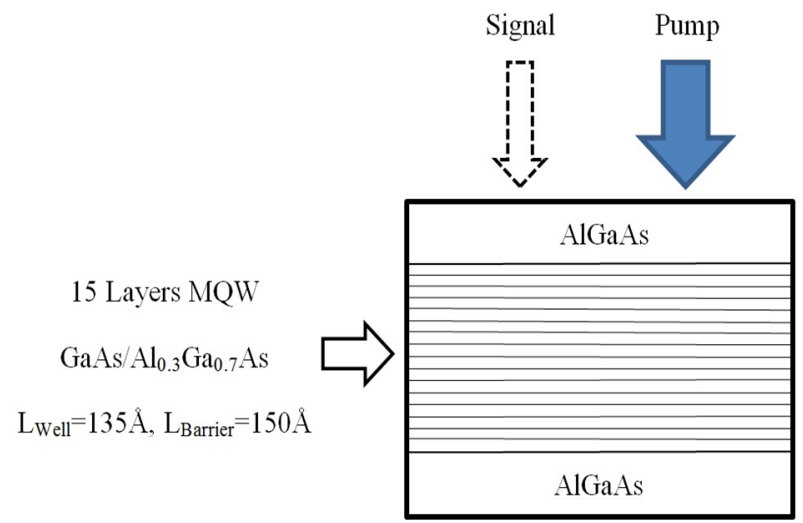

Figure 1. MQW in presence of pump and signal for slow light purpose.
For investigation the effect of exciton oscillator strength (EOS) and binding energy on slow light device, three experiments in GaAs/AlGaAs multiple quantum wells were considered. The first experiment is about slow light using excitonic population [1]. The second experiment introduces the dependence of EOS on quantum well width [10-11]. The last one, considers the effects of QW size on binding energy of excitons [12]. It is worth mentioning that this topology was selected as a test bench and the results of analyses can easily be used in other implementations of slow light devices as well.

\subsection{Dependence of EOS on Quantum Well Width}

Absorption of excitons can be described in terms of $\varepsilon_{e x}$ (dielectric constant) as follows [9]:

$$
\varepsilon_{e x}=\frac{A}{E_{e x}^{2}-E^{2} i E \Gamma}=\varepsilon_{e x r}+\varepsilon_{e x i}
$$

where $\varepsilon_{e x r}$ and $\varepsilon_{e x i}$ are respectively the real and imaginary parts of $\varepsilon_{e x}, E_{e x}$ is the transition energy of excitons, $\Gamma$ and $E$ are linewidth (FWHM) and the photon energy, respectively. Parameter $A$ is related to oscillator strength per unit area. Absorption coefficient $\alpha$ is related to oscillator strength per unit area $f$ by [13]:

$$
\alpha=\frac{4 \pi^{2} e^{2} \hbar f}{n m_{0} c L} \Delta\left(E-E_{e x}\right)
$$

where

$$
\Delta\left(E-E_{e x}\right)=\frac{\Gamma}{2 \pi} \frac{1}{\left(E-E_{e x}\right)^{2}+(\Gamma / 2)^{2}}
$$

where $m_{0}$ is the free electron mass and $L$ is the well width. $c$ is the light velocity in vacuum and $n$ is refractive index of the material. The oscillator strength per unit area can be obtained as follow by comparing equations (1) and (2):

$$
f=\frac{m_{0} A L}{8 \pi \hbar^{2} e^{2}}
$$

According to [11], for narrow wells, the factor $\mathrm{A}$ is directly proportional to oscillator strength, $f$, and related to well width as $A \approx 1 / L^{2}$. As a result, the EOS is related to width of quantum well inversely.

Figure 2 shows the behavior of EOS for ground state HH1-CB1 according to quantum well width. The parameters which are used to simulate are the same with [10-11].

\subsection{Dependence of Binding Energy on Quantum Well Width}

The exciton bound-state energies and wave functions as a function of spatial dimension calculated according to $\mathrm{He}$ 


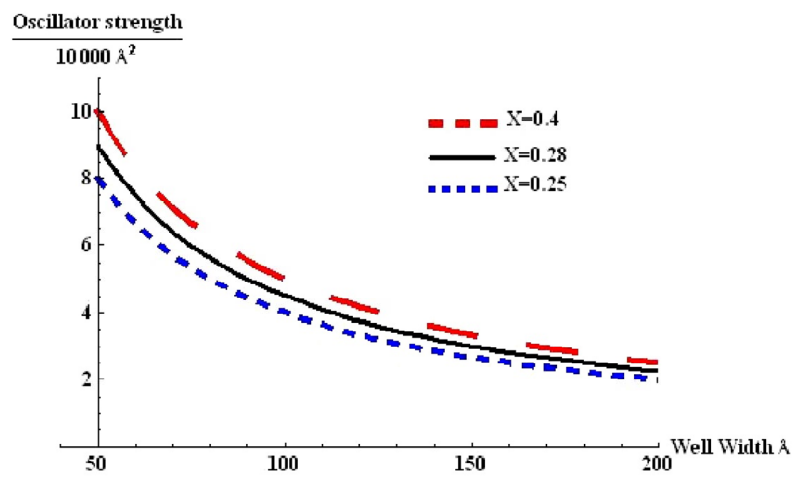

Figure 2. Well width dependence of the excitonic oscillator strength in $G a A s / A l_{x} G a_{(1-x)} A s$ with different alloy percentage $x$.

model [14]. The discrete bound-state energies and orbital radii are given by [14]:

$$
\begin{gathered}
E_{n}=E_{g}-\frac{E_{0}}{\left(n+\frac{\alpha-3}{2}\right)} \\
\alpha_{n}=\alpha_{0}\left(n+\frac{\alpha-3}{2}\right)
\end{gathered}
$$

where $n$ is quantum number, $E_{0}$ and $\alpha_{0}$ are effective Rydberg constant, $\left(\left(\varepsilon_{0} / \varepsilon\right)^{2}\left(\mu / m_{0}\right)\right) R_{H}$, and effective Bohr radius, $\left(\left(\varepsilon / \varepsilon_{0}\right)\left(m_{0} / \mu\right)\right) \alpha_{H}$, respectively. $R_{H}$ and $\alpha_{H}$ are the Rydberg and Bohr radius constant, $m_{0}$ is the free electron mass, $\mu$ is exciton reduced mass, $\varepsilon$ is dielectric constant.

According to Equation (5), the binding energy of the $1 \mathrm{~s}$ exciton is given by:

$$
E_{b}=\left(\frac{2}{\alpha-1}\right)^{2} E_{0} S
$$

$\alpha$ changes between 2 and 3 in real quantum well structure. Reduction in well width causes compression in envelope function of electron and hole pairs.

Consequently the fractional dimension $\alpha$ decreases from 3 towards 2 [12]. Although in a very narrow wells because of spreading of envelope function in barrier level the spatial extents begin to increase as the well width decreases.

According to Figure 1 which describes the geometry of a slow light device, the well width of QW is finite. When the well width decreases below a specific value, the envelope function spread into barriers. This phenomenon restores the three dimensional character of excitons. As a result, fractional dimension $\alpha$ should be expressed as a function of spreading of envelop function. As a result the fractional dimension $\alpha$ is described as follows [12]:

$$
\alpha=3-e^{2\left(2 / k_{b}+L_{w}\right) / 2 \alpha^{*}}
$$

In above equation $k_{b}$ and $\alpha_{0}^{*}$ are respectively, the wave vector in barrier and the mean value of the three dimensional Bohr-radius which is defined as follows:

$$
\alpha_{0}^{*}=\frac{\varepsilon}{\varepsilon_{0}} \frac{m_{0}}{\mu} \alpha_{H}
$$

where $\mu$ is a mean value of the three dimensional reduced mass of exciton. The binding energy of the combined exciton in a finite quantum well is defined as follows [12]:

$$
E_{b}=\frac{E_{0}^{*}}{\left(1-0.5 e^{-\left(2 / k_{b}+L_{w}\right) / 2 \alpha_{0}^{*}}\right)^{2}}
$$

where $E_{0}^{*}$ is the mean value of the effective Rydberg energy for the three dimensional exciton. The details of solutions and the exact definition of parameters can be found in [12].

Figure 3 shows the behavior of binding energy for ground state $\mathrm{HH} 1-\mathrm{CB} 1$ according to quantum well width. The parameters which are used to simulate are the same with [12-14]. It is important to note that Figure 3 does not show the binding energy under $50 \AA$ well width. Under $50 \AA$, the behavior of binding energy will change and because of spreading the envelop function in barrier level; the amount of binding energy will decrease. However in this paper, we don't consider this phenomenon because in quantum well slow light device the width of quantum well is more than $50 \AA$.

\subsection{Slow Light Using Excitonic Population Oscillation}

In this subsection, the primary attention is description of general theory of slow light in semiconductors. "Equation (11) shows the group velocity of an optical pulse in a medium with refractive index $n(\omega)$ [4-5]".

$$
v_{g}=\frac{c}{n(\omega)+\omega \frac{d n(\omega)}{d \omega}}=\frac{c}{n_{g}}
$$

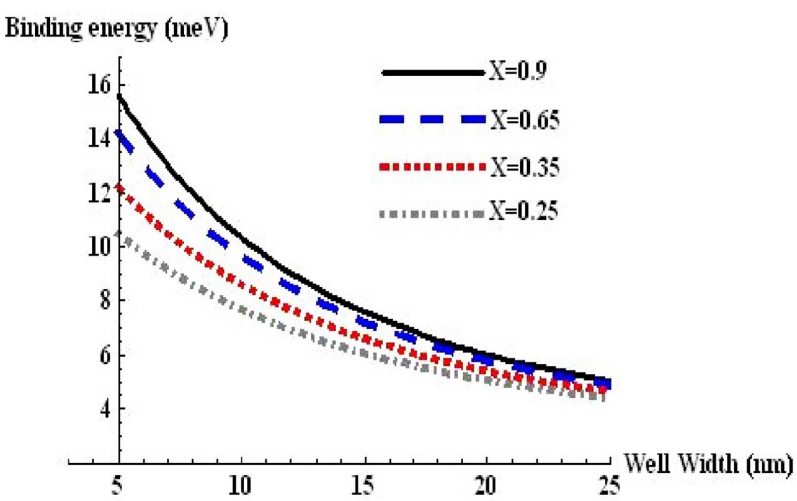

Figure 3. Well width dependence of the binding energy in $G a A s / A I_{x} G a_{(1-x)} A s$ with different alloy percentage $x$. 
Slow light in semiconductor can be obtained by increasing the refractive index. One possible way for reducing group velocity is using materials in which $d n / d \omega$ is as large as possible [14]. The refractive index of such sample is typically small [15-16]. But it varies rapidly in the region of the resonance so $d n / d \omega$ and hence $n_{g}$ can be large. However, the absorption near resonance is very high. So, all of light is absorbed in the length of the device [16]. But, the effect of absorption is canceled by using coherent optical effect. Laser beam called pump laser, is tuned to one of the resonance frequency of material. It causes a narrow dip in absorption in profile [17].

In materials with saturable absorption, when a strong pump and a signal beam of slightly different frequencies interact, the population of ground state will oscillate in time at the beat frequency of two input beam. This phenomenon is called coherent population oscillation [16].

In the rest of this section, we consider a slow light system with excitonic population oscillation which is described in [1]. In a simple two level system, the coherent population beating induced by the frequency difference between the signal and the pump can produce a dip in the absorption spectrum if dephasing time is much shorter than the relaxation time of the population difference [18]. For investigating of dispersion in such system, usually semiconductor Bloch equations is used. Moreover, only the first $\mathrm{C}$-like and $\mathrm{HH}$-like quantized bands are taken into consideration [15]. Table 1 shows the definitions of parameters which are used in this subsection.

The coherent dynamic equations for Interband polarization and carrier occupation numbers are as follows [1]:

$$
\begin{aligned}
\frac{\partial P_{e x, \sigma}}{\partial t}= & -i\left[\omega_{e x}-i \Gamma_{2}\left(N_{e x}\right)\right] P_{e x, \sigma}-i \frac{\mu_{12} \varepsilon(t)}{2 \hbar} N_{e x, \sigma} \\
\frac{\partial N_{e x, \sigma}}{\partial t}= & -\Gamma_{1}\left(N_{e x, \sigma}-N_{e x, \sigma}^{(0)}\right)-\Gamma_{s}\left(N_{e x, \sigma}-N_{e x, \ddot{\sigma}}\right) \\
& +4 \operatorname{Im}\left[\frac{\mu_{12} \varepsilon(t)}{2 \hbar} P_{e x, \sigma}\right]
\end{aligned}
$$

where $N_{e x, \sigma}^{(0)}$ is the respective population difference in equilibrium. This equation, (12), is valid only in low excitation regime. In this approximation the effect of electron hole plasma screening and phase space filling are not include and the equation can be solved in the steady state region. With relevant optical equation in semiconductor, the linear relative permittivity tensor $\varepsilon_{s}\left(\omega_{s}\right)$ experienced by the signal is obtained. Refractive index, absorption and slow down factor are defined respectively as follows:

$$
\begin{gathered}
n_{s}\left(\omega_{s}\right)=\sqrt{\varepsilon_{s}\left(\omega_{s}\right)} \\
A_{s}\left(\omega_{s}\right)=2 \frac{\omega_{s}}{c} \operatorname{Im}\left[n_{s}\left(\omega_{s}\right)\right]
\end{gathered}
$$

$$
R_{s}\left(\omega_{s}\right)=\operatorname{Re}\left[n_{s}\left(\omega_{s}\right)\right]+\omega_{s} \frac{\partial \operatorname{Re}\left[n_{s}\left(\omega_{s}\right)\right]}{\partial \omega_{s}}
$$

Table 2 shows the experimental parameters which is used in [1-15] for calculations. According to the absorption coefficient and real part of refractive index are shown in [19], for resonance frequency the value of $d n / d \omega$ is high. So, the group index increases rapidly and the effect of slow light can be seen in device.

\section{Modified Fourier Transform Spectral Interferometry Model for Slow Light Device}

For modeling a slow light device, the model which is described in [20] is used. This model gives an analytical expression for the full dielectric constant of Wannier exciton of arbitrary dimensionality, $d,[21]$. The effective dimensionality $d$, is a parameter that can vary between 2 and 3 in quantum well, depending on the ratio of the QW thickness to the exciton Bohr radius. Moreover, $d$ is also related to the spatial extension of the exciton in the barriers. The complex dielectric constant according to this model is [20]:

Table 1. Definition of the symbols.

\begin{tabular}{cc}
\hline Symbol & Definition \\
\hline$P_{e x}$ & $\begin{array}{c}\text { Interband polarization } \\
\text { Effective population } \\
\text { difference }\end{array}$ \\
\hline$N_{e x}$ & \begin{tabular}{c} 
Excitonic frequency \\
\hline$\omega_{e x}$
\end{tabular} \\
\hline$\sigma$ & $\begin{array}{c}\text { Spin index } \\
\text { Relaxation constant }\end{array}$ \\
$\Gamma_{1}$ & Spin flip constant \\
\hline$\Gamma_{S}$ & Polarization dephasing \\
\hline$\Gamma_{2}\left(N_{e x}\right)$ & $\begin{array}{c}\text { Excitation inchiced } \\
\text { dephasing }\end{array}$ \\
\hline$(E I D)$ & $\begin{array}{c}\text { Rabi frequency } \\
\left(\Omega=\mu_{12} E / 2 \hbar\right)\end{array}$ \\
\hline$\Omega$ & $\begin{array}{c}\text { Dipole momentum } \\
\text { between }|1>,| 2>\end{array}$ \\
\hline$\mu_{12}$
\end{tabular}

Table (2). Physical device parameter for $G a A s / A I_{0.3} G a_{0.7} A s$.

\begin{tabular}{cc}
\hline Parameter & Value \\
\hline $\begin{array}{c}\text { Quantum well effective } \\
\text { width number }\end{array}$ & $2025 \AA / 15$ \\
\hline $\begin{array}{c}\text { Excitonic energy } \\
\text { (Pump energy) }\end{array}$ & $1.5535 \mathrm{eV}$ \\
\hline $\begin{array}{c}\text { Variation of } 1 \mathrm{~s} \\
\text { excitonic in plane wave } \\
\text { Relaxation constant }\end{array}$ & $3.18 \times 10^{5} \AA^{-2}$ \\
\hline Spin flip constant & $2.5133 \mathrm{~ns}^{-1}$ \\
\hline Dephasing constant & $0.4716 \mathrm{ps}^{-1}$ \\
\hline EID & $2.24 \mathrm{ps}^{-1}$ \\
\hline E back ground & 12.25 \\
\hline
\end{tabular}




$$
\begin{aligned}
\varepsilon(E)= & \frac{S \times R^{0.5 d-1}}{(E+i \gamma)}\left(g_{d}[\xi(E+i \gamma)]+g_{d}[\xi(E+i \gamma)]\right. \\
& \left.-2 g_{d}[\xi(0)]\right)
\end{aligned}
$$

where

$$
\begin{aligned}
g_{d}(\xi)= & \frac{2 \pi \Gamma\left(\frac{d-1}{2}+\xi\right)}{\Gamma\left(\frac{d-1}{2}\right)^{2} \Gamma\left(1-\frac{d-1}{2}+\xi\right) \xi^{d-2}} \\
& \times\left(\cot \left[\pi\left(\frac{d-1}{2}-\xi\right)\right]-\cot [\pi(d-1)]\right)
\end{aligned}
$$

where $\Gamma(x)$ is Euler gamma function, $R$ and $E_{g}$ are the effective Rydberg and band gap energy, $\gamma$ is linewidth, and $S$ is parameter which is related to exciton oscillator strength. The function $\xi(z)$ is defined as, $\xi(z)=\left(R /\left(E_{g}-z\right)\right)^{0.5}$.

Considering the heavy and light hole, the total dielectric constant for the QW is defined as follows [20]:

$$
\varepsilon(E)=1+\varepsilon_{\text {background }}+\varepsilon_{\text {heavy }}(E)+\varepsilon_{\text {light }}(E) .
$$

It is worthwhile to note that in the case of excitonic population oscillation for slow light purpose, only the heavy hole contribution is considered.

Since we want to investigate the effect of oscillator strength on slow light device, we changed the sign of dielectric constant. Because of pump signal configuration in quantum well and absorption cancellation, the steep direction of refractive index near resonance frequency is changed. So we have to change the sign of dielectric constant to use this model for slow light devices. Base on this modified model, the refractive index profile for QW slow light device with different oscillator strength is shown in Figure 4. EOS is change with aim of reducing or increasing QW width. As it is clear from this figure, increasing the oscillator strength will cause improvement in steep of refractive index.

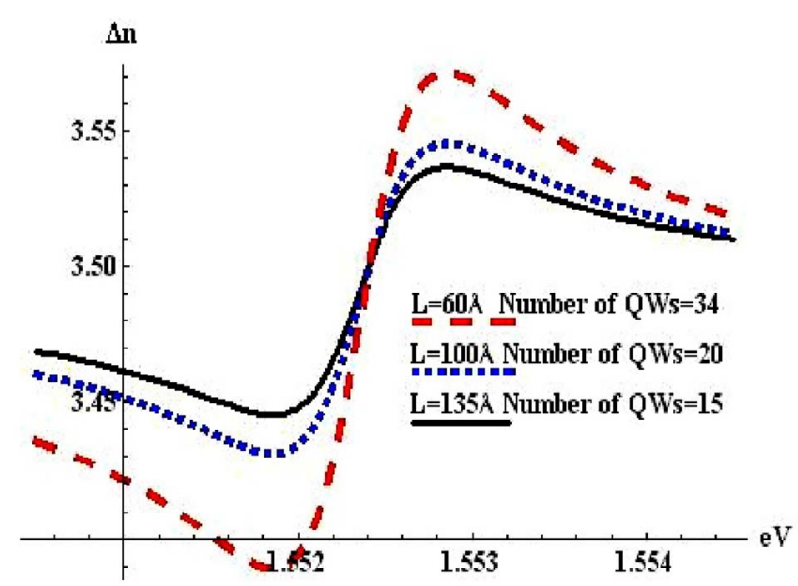

Figure 4. The refractive index profile for $\mathrm{QW}$ slow light device with different oscillator strength. The solid line shows experimental result of [1].
In Figure 4, the parameters values are $d=2.5, R_{H}=$ $4.3 \mathrm{meV}, E_{g}=1.56 \mathrm{eV}, \gamma=0.5 \mathrm{meV}$, according to [20-22]. On the other hand according to "Equation (11)", slow down factor is directly proportional to derivative of refractive index. As a result, increasing the EOS will cause enhancement in slow down factor.

\section{Results and Discussion}

\subsection{Binding Energy Effects}

According to mentioned theory in section (2.2), the binding energy will increase if the size of quantum well decreases. "Equation (19) shows the dependence of excitonic energy to binding energy". This equation shows that if binding energy is increased, the excitonic energy will be decreased.

$$
E_{e x}=E_{\text {band-gap }}-E_{\text {binding-energy }}
$$

Excitonic energy presents the center frequency of slow light device base on excitonic population oscillation. As a result, the size of quantum well in slow light device can shift center frequency of device. This shift is upward when the well width is increased and downward when well width is decreased. With aim of this phenomenon, we can tune the center frequency of our slow light device.

To verify the effect of quantum well size on center frequency of device, we simulate the experimental device which is presented in section (2.3) with different well width sizes. The parameters for simulation are extracted from [1-10-12].

Figure 5 shows the refractive index of a quantum well slow light device with three different well width sizes. As it is clear from this figure, the resonance frequency of $G a A s / A l_{0.3} G a_{0.7} A s$ QWs is shift with changing well size. The refractive index profile broadening in Figure $\mathbf{5}$ is

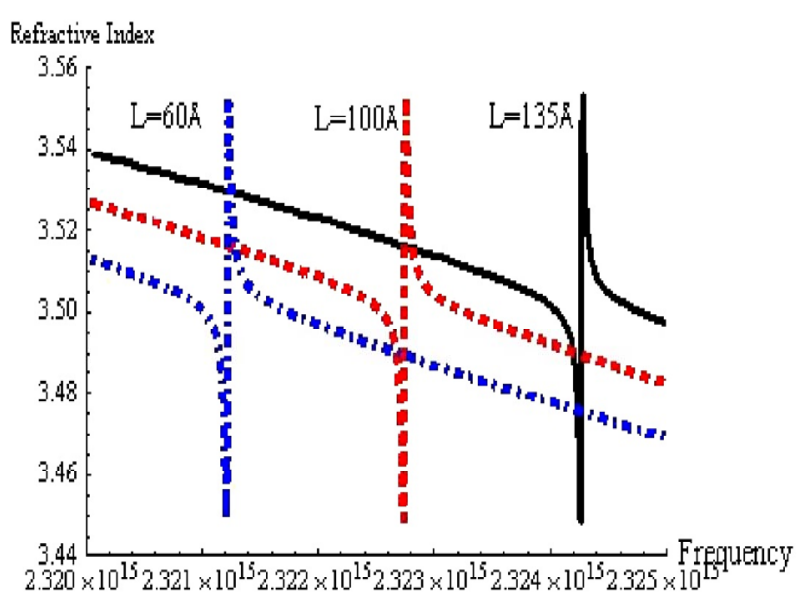

Figure 5. The refractive index profile for $\mathrm{QW}$ slow light device with different binding energy. The solid line shows experimental result of [1]. 
very narrow because we want to show these three cases in one coordinate. Beside, in Figure 5, the effect of QW size on EOS is neglected. As far as we know, near resonance frequency the derivative term in "Equation (11)" increases rapidly and causes huge slow down factor. Moreover, the transparency window is created which causes cancellation of absorption effect. The size of well width determines the location of transparency window in frequency domain.

Table 3 shows the frequency shift and center of a slow light with three different well widths. Experimental well size of [1] is considered as origin. It is important to note that there is obviously limitation to reducing QW size. As mentioned in previous section, under specific well width size which is about $50 \AA$ the behavior of binding energy changes and other phenomenon happens.

Base on simulation results of this subsection, the center frequency for QW slow light device will decrease if the well width of quantum well decreases and the number of QWs increases. The number of QWs must be increased because the effective QWs width as mentioned in Table 2 should be constant. For example if the well width is divided by factor 2 , then the number of QWs must be increased by the same factor.

\subsection{Exciton Oscillator Strength Effects}

According to theory (2.1), we will use the effect of decreasing length of quantum well as parameter for increasing the EOS.

Base on simulation results of section (3), the slow down factor for QW slow light device will increase if the well width of quantum well decreases and the number of QWs increases. The number of QWs must be increased based on the same reason which mentioned above. To show the usefulness of the proposed method, the $G a A s / A l_{0.3}$ $G a_{0.7} A s$ multiple quantum wells shown in Figure 1 was simulated. But this time, we consider the effect of reducing QWs width.

Figure 6 shows the slow down factor for three different QWs width. It can be seen that decreasing the well width will increase the slow down factor.

\section{Conclusions}

We investigate the effects of excitonic oscillator strength and binding energy of exciton on slow light devices.

Table 3. Frequency shift according to well width size.

\begin{tabular}{cccc}
\hline $\begin{array}{c}\text { Well Width } \\
\text { Size }\end{array}$ & $135 A$ (Origin) & $100 A$ & $60 A$ \\
\hline $\begin{array}{c}\text { Center } \\
\text { Frequency } \\
(\mathrm{Hz})\end{array}$ & $2.32426 \times 10^{15}$ & $2.32274 \times 10^{15}$ & $2.32121 \times 10^{15}$ \\
\hline $\begin{array}{c}\text { Frequency } \\
\text { Shift }(\mathrm{Hz})\end{array}$ & --- & $1.5238 \times 10^{12}$ & $3.0476 \times 10^{12}$ \\
\hline
\end{tabular}

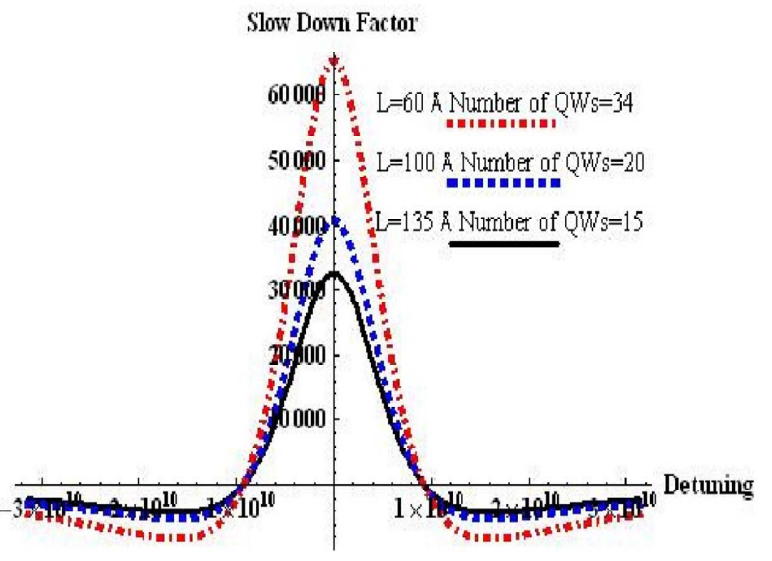

Figure 6. The slow down factor for three different QWs width. The solid line shows experimental result of [1].

According to described model in section (3), the slow down factor can be increased to more than 60,000 in the result of reducing well width. Moreover, the center frequency of transparency window can be shift about $3.0476 \times 10^{12} \mathrm{~Hz}$ with aim of binding energy. When QW size is changed, both oscillator strength and binding energy of excitons are affected. Therefore, when the device experiences downshift or upshift in its transparency window, in the same time, the amount of slow down factor is increased or decreased respectively. Based on these investigations, the new method for tuning slow light properties is developed. This novel technique will help designer to control properties of device. As far as we know, semiconductor slow light devices suffer from narrow bandwidth. Moreover there are huge uses of multichannel system for slow light devices to overcome limited bandwidth problem. Multichannel systems need slow light devices with different center frequencies. With the aim of this technique, designers could fabricate devices with different center frequencies and slow down factor. Because of availability of center frequency shifting and SDF controlling in this method, we could tune our slow light device for optimal operational point. In addition, this availability of frequency shifting in slow light device could be interpreted as increasing of operational bandwidth.

\section{REFERENCES}

[1] S. W. Chang, S. L. Chuang, P. C. Ku, C. J. Chang-Hasnia n, P. Palinginis and H. L. Wang, "Slow Light Using Ex citonic Population Oscillation," Physical Review B, Voljhjklkjh kj. 70, No. 23, 2004. doi:10.1103/PhysRevB.70.235333

[2] D. Sun and P. C. Ku, "Slow Light Using P-Doped Semiconductor Heterostructures for High-Band-Width Nonlinear Signal Processing," Journal of Lightwave Technology, Vol. 26, No. 23, 2008, pp. 3811-3817. doi:10.1109/JLT.2008.2005121 
[3] E. Parra and J. R. Lowell, "Toward Applications of Slow Light Technology," Optics and Photonics News, Vol. 18, 2007, pp. 40-45. doi:10.1364/OPN.18.11.000040

[4] M. M. Kash, V. A. Sautenkov, A. S. Zibrov, L. Hollberg, G. R. Welch, M. D. Lukin, Y. Rostovtsev, E. S. Fry and M. O. Scully, "Ultraslowgroup Velocity and Enhanced Nonlinear Optical Effects in A Coherently Driven Hot Atomic Gas," Physical Review Letters, Vol. 82, 1999, pp. 5229.doi:10.1103/PhysRevLett.82.5229

[5] B. Pesala, Z. Y. Chen, A. V. Uskov and C. Chang-Hasnain, "Experimental Demonstration of Slow and Superluminal Light in Semiconductor Optical Amplifiers," Optics Express, Vol. 14, 2006, pp. 12968-12975. doi:10.1364/OE.14.012968

[6] Y. Okawachi, M. A. Foster, J. E. Sharping, A. L. Gaeta, Q. $\mathrm{Xu}$ and M. Lipson, "All Optical Slow-Light on A Photonic Chip," Optics Express, Vol. 14, 2006, pp. 2317-2322.doi:10.1364/OE.14.002317

[7] Haug and S. W. Koch, "Quantum Theory of the Optical and Electronic Properties of Semiconductors," 3rd Edition, World Scientific, Singapore, 1994.

[8] C. J. Chang-Hasnain, P. C. Ku, J. Kim and S. L. Chuang, "Variable Optical Buffer Using Slow Light in Semiconductor Nanostructures," Proceedings of the IEEE, Vol. 91, No. 11, 2003, pp. 1884-1897. doi:10.1109/JPROC.2003.818335

[9] G. P. Agrawal, "Population Pulsations and Nondegenerate Four-Wave Mixing in Semiconductor Lasers and Amplifiers," Journal of the Optical Society of America B, Vol. 5, No. 1, 1988, pp. 147-159. doi:10.1364/JOSAB.5.000147

[10] B. P. Zhang, S. S. Kano and Y. Shiraki, "Reflectance Study of the Oscillator Strength of Ecitons in Semiconductor Quantum Wells," Physical Review B, Vol. 50, 1994, pp. 7499-7508.doi:10.1103/PhysRevB.50.7499

[11] W. T. Masselink, P. J. Pearah, J. Klem, C. K. Peng and H. Morkoç, "Absorption Coefficients and Exciton Oscillator Strengths in AlGaAs-GaAs Superlattices," Physical Review $B$, Vol. 32, 1985, pp. 8027-8034. doi:10.1103/PhysRevB.32.8027

[12] H. Mathieu, P. Lefebvre and P. Christol, "Simple Ana- lytical Method for Calculating Exciton Binding Energies in Semiconductor Quantum Wells," Physical Review B, Vol. 46, 1992, pp. 4092-4101. doi:10.1103/PhysRevB.46.4092

[13] E. Parra and J. R. Lowell, "Toward Applications of Slow Light Technology," Optics and Photonics News, Vol. 18, 2007, pp. 40-45. doi:10.1364/OPN.18.11.000040

[14] X. F. He, "Excitons in Anisotropic Solids: The Model of Fractional- Dimensional Space," Physical Review B, Vol. 43, 1991, pp. 2063-2069.doi:10.1103/PhysRevB.43.2063

[15] R. S. Knox, "Theory of Excitons," Academic Press, New York and London, 1963.

[16] S. W. Chang and S. L. Chuang, "Slow Light Based on Population Oscillation in Quantum Dots with Inhomogeneous Broadening," Physical Review B, Vol. 72, No. 23, 2005, pp. 235330. doi:10.1103/PhysRevB.72.235330

[17] C. J. Chang-Hasnain and S. L. Chuang, "Slow and fast light in semiconductor quantum well and quantum-dot devices," Journal of Lightwave Technology, Vol. 24, No. 12, 2006, pp. 4642-4654. doi:10.1109/JLT.2006.885767

[18] M. Sargent III, "Spectroscopic Techniques Based on Lamb's Laser Theory," Physics Reports, Vol. 43, No. 5, 1978, pp. 223-265. doi:10.1016/0370-1573(78)90163-1

[19] H. Kaatuzian, H. S. Kojori and M. Danaei, 7th Int. Symp. High-Capa. Opt. Net. Enb. Tech. (HONET), Cairo, Egypt, 2010, p. 143.

[20] M. V. Marquezini, J. Tignon, T. Hasche and D. S. Chemla, "Refractive Index and Absorption of GaAs Quantum Wells Across Excitonic Resonances," Applied Physics Letters, Vol. 73, 1998, pp. 2313. doi: $10.1063 / 1.121808$

[21] C. Tanguy, P. Lefebvre, H. Mathieu and R. J. Elliott, "Analytical Model for the Refractive Index in Quantum Wells Derived from the Complex Dielectric Constant of Wannier Excitons in Noninteger Dimensions," Journal of Applied Physics, Vol. 82, 1997, pp. 798. doi:10.1063/1.365580

[22] L. C. Andreani and A. Pasquarello, "Accurate Theory of Exciton in GaAs-GaAlAs Quantum Well," Physical Review $B$, Vol. 42, 1990, pp. 8928. doi:10.1103/PhysRevB.42.8928 